\title{
Gap suppression at a Lifshitz transition in a multi-condensate
}

\section{superconductor}

\author{
G. Singh ${ }^{1,2}$, A. Jouan ${ }^{1,2}$, G. Herranz ${ }^{3}$, M. Scigaj ${ }^{3}$, F. Sánchez ${ }^{3}$, L. Benfatto ${ }^{4,5}$, S. Caprara ${ }^{5,4}$, \\ M. Grilli ${ }^{5,4}$, G. Saiz ${ }^{1,2}$, F. Couedo ${ }^{1,2}$, C. Feuillet-Palma ${ }^{1,2}$, J. Lesueur ${ }^{1,2}$, N. Bergeal ${ }^{1,2 *}$ \\ ${ }^{1}$ Laboratoire de Physique et d'Etude des Matériaux, \\ ESPCI Paris, PSL Research University, CNRS, \\ 10 Rue Vauquelin - 75005 Paris, France. \\ ${ }^{2}$ Université Pierre and Marie Curie, \\ Sorbonne-Universités, 75005 Paris, France. \\ ${ }^{3}$ Institut de Ciéncia de Materials de Barcelona (ICMAB-CSIC), \\ Campus de la UAB, 08193 Bellaterra, Catalonia, \\ Spain. ${ }^{4}$ Institute for Complex Systems (ISC-CNR), \\ UOS Sapienza, Piazzale A. Moro 5, 00185 Roma, Italy \\ ${ }^{5}$ Dipartimento di Fisica Università di Roma "La Sapienza", \\ Piazzale A. Moro 5, I-00185 Roma, Italy.
}

In multi-orbital materials, superconductivity can exhibit several coupled condensates. In this context, quantum confinement in two-dimensional superconducting oxide interfaces offers new degrees of freedom to engineer the band structure and selectively control 3d-orbitals occupancy by electrostatic doping. Here, we use resonant microwave transport to extract the superfluid stiffness of the (110)-oriented $\mathrm{LaAlO}_{3} / \mathrm{SrTiO}_{3}$ interface in the entire phase diagram. We evidence a transition from single-condensate to two-condensate superconductivity driven by continuous and reversible electrostatic doping, which we relate to the Lifshitz transition between 3d-bands based on numerical simulations of the quantum well. We find that the superconducting gap is suppressed while the second band is populated, challenging the Bardeen-Cooper-Schrieffer theory. We ascribe this behavior to the existence of superconducting order parameters with opposite signs in the two condensates, due to a repulsive coupling. Our findings offer an innovative perspective on the possibility to tune and control multiple-orbitals physics in superconducting interfaces.

In cubic perovskites, transition metal ions are surrounded by six oxygen ions in an octahedron configuration. Because of the negative charge of $\mathrm{O}^{2-}$ ions, the transition metal $3 d$ electrons are subject to an anisotropic crystal field, which splits the five $3 d$ orbitals into three degenerated $t_{2 g}$ 
orbitals $\left(d_{x y}, d_{x z}\right.$ and $\left.d_{y z}\right)$ and two degenerated $e_{g}$ orbitals $\left(d_{x^{2}-y^{2}}\right.$ and $\left.d_{z^{2}}\right)$ at higher energy [1]. This situation is encountered in bulk $\mathrm{SrTiO}_{3}$ crystals whose conduction band is formed by the coupling between $t_{2 g}$ orbitals at neighbouring Ti lattice sites through the $2 p$ orbitals of the oxygen atoms. Meanwhile, the physical properties of two-dimensional $\mathrm{SrTiO}_{3}$-based superconducting interfaces such as $\mathrm{LaAlO}_{3} / \mathrm{SrTiO}_{3}[2,3]$ or $\mathrm{LaTiO}_{3} / \mathrm{SrTiO}_{3}$ [4] interfaces are also deeply affected by the further splitting of the $t_{2 g}$ bands under quantum confinement. Many theoretical works have shown that a complex band structure involving bands with different orbital symmetries emerges at the interface [5-9]. These predictions are supported by experiments such as x-ray absorption spectroscopy [10], optical conductivity [11], Hall effect [12-14] and quantum oscillations [15-17], which evidence multiband transport at high carrier densities. The presence of several disconnected Fermi surface sheets makes these materials more prone to exhibit unconventional properties.

In the conventional weak-coupling BCS theory, the superconducting critical temperature is expressed as $T_{c} \simeq \hbar \omega_{D} e^{-\frac{1}{\lambda}}$, where $\hbar \omega_{D}$ is an energy cutoff (Debye energy in the standard phononmediated pairing mechanism) and $\lambda=N(0) V_{0}$ is the coupling constant [18]. Because of the exponential factor, the density of states at the Fermi level, $N(0)$ and the pairing potential, $V_{0}$, are the most relevant parameters in the determination of $T_{c}$. Although the origin of superconductivity in $\mathrm{SrTiO}_{3}$ is still debated, it is widely believed that its gigantic low-temperature dielectric constant $\epsilon_{R} \simeq 25000$ [19] should play a pivotal role in the pairing mechanism and consequently in the determination of $V_{0}$. On the other hand, in a two-dimensional system, the density of states is directly proportional to the effective mass $m^{*}$ of the carriers, $N(0)=\frac{m^{*}}{\pi \hbar^{2}}$. One important feature of $\mathrm{SrTiO}_{3}$ - based interfaces lies in the possibility of controlling the filling of a set of bands having different properties, e. g. $V_{0}$ and $N(0)$, by electrostatic gating [20-22]. Several attempts have been made to relate the emergence of superconductivity to the different band occupancies [20, 22]. However, only one superconducting gap has been reported in the conventional (001)-oriented interfaces $[22,24,25]$. Here, we use resonant microwave transport to investigate the superfluid stiffness of the (110)-oriented interface. We evidence a gate-driven transition from single-condensate to two-condensate superconductivity, which has never been reported so far in superconductors. At high carrier doping, we relate the two condensates to the degenerated $d_{x z} / d_{y z}$ bands on the one hand and the $d_{x y}$ band on the other hand. Because of the unusual decrease of $T_{c}$ concomitant with the emergence of the second gap, we propose that the two conden-

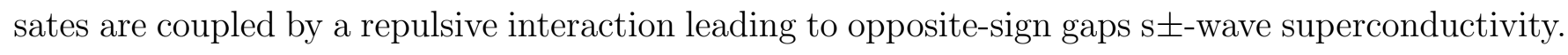

While most studies have focused on (001)-oriented $\mathrm{LaAlO}_{3} / \mathrm{SrTiO}_{3}$ heterostructures, it has been shown that a superconducting two-dimensional electron gas (2-DEG) can also develop in other 

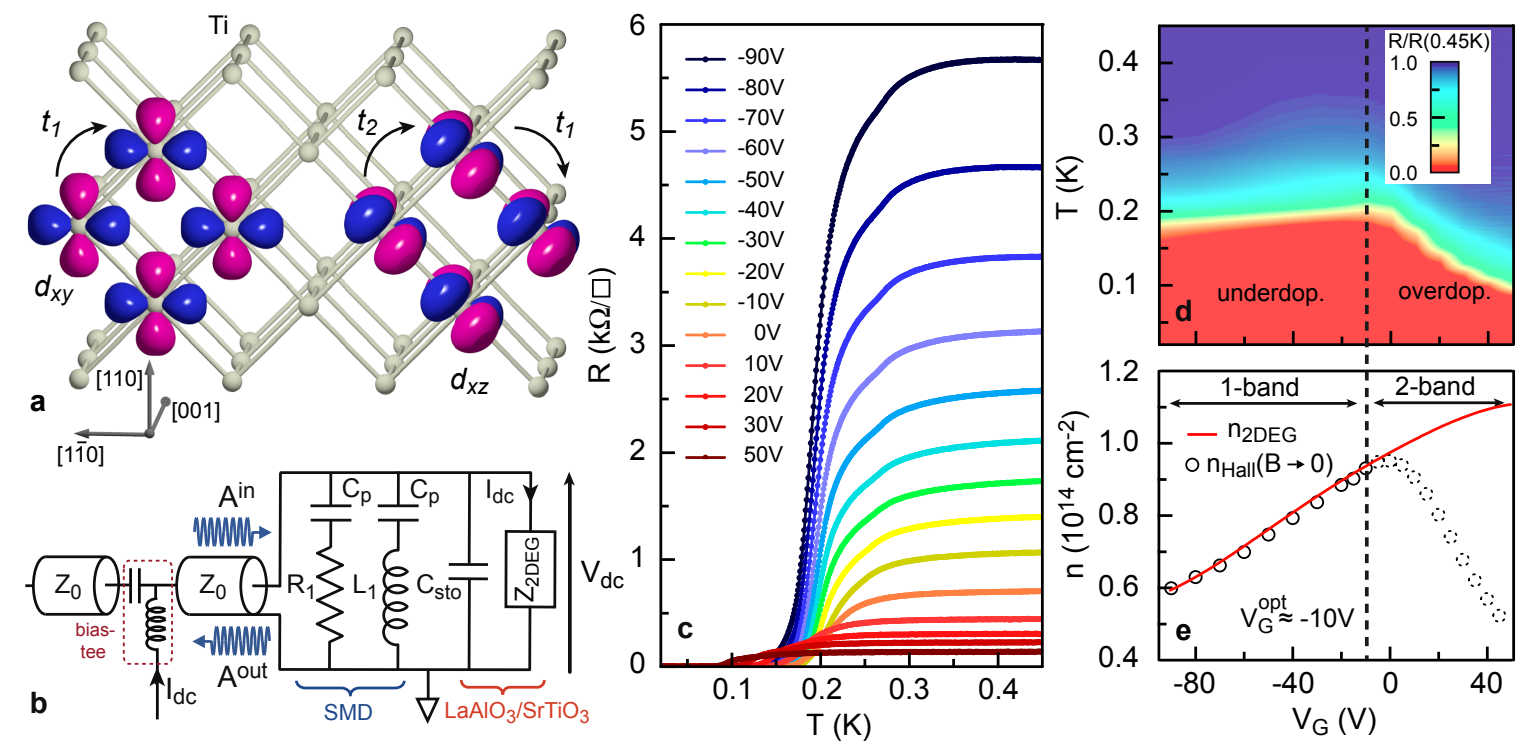

Figure 1: Superconductivity and multiband transport in (110)-oriented $\mathrm{LaAlO}_{3} / \mathrm{SrTiO}_{3}$ interfaces. (a) Schematic of the $t_{2 g}$ orbitals of $\mathrm{Ti}$ atoms in the (110)-oriented $\mathrm{SrTiO}_{3}$. The positive and negative lobes of the orbitals are represented by two different colors (purple and blue). $d_{y z}$ orbitals (not shown) are obtained by a $90^{\circ}$ rotation of the $d_{x z}$ ones along the (001) direction. $t_{1}$ and $t_{2}$ represent the hopping terms along the (001) and (1-10) directions of the interface plane. In this experiment current was injected along the (1-10) direction. (b) Resonant sample circuit comprising the $\mathrm{LaAlO}_{3} / \mathrm{SrTiO}_{3}$ heterostructure represented by the 2-DEG impedance $Z_{2 \mathrm{DEG}}$ and the intrinsic $\mathrm{SrTiO}_{3}$ capacitance $C_{\text {sto }}$, and the Surface Mounted Devices (SMD), resistor $R_{1}=100 \Omega$ and inductor $L_{1}=9 \mathrm{nH} . C_{p}=2 \mu \mathrm{F}$ are protective capacitances that avoid dc current to flow through $L_{1}$ and $R_{1}$ without influencing the resonance frequency of the circuit $\omega_{0}$ [22]. A bias-tee is used to separate the dc signal from the microwave one. $\mathrm{A}^{\text {in }}$ and $\mathrm{A}^{\text {out }}$ are the complex amplitudes of the incident and the reflected microwave signals. (c) Sheet resistance as a function of temperature measured in dc for different gate voltages in the range $[-90 \mathrm{~V},+50]$. (d) Sheet resistance normalized by its value at $\mathrm{T}=0.45 \mathrm{~K}$ in color scale as a function of gate voltage and temperature. (e) Hall carrier density in the limit of zero-magnetic field $B, n_{\text {Hall }}(B \rightarrow 0)$ (circles), measured at $T=3 \mathrm{~K}$ as a function of $V_{\mathrm{G}} . V_{\mathrm{G}}^{\mathrm{opt}} \simeq-10 \mathrm{~V}$ is the optimal doping corresponding to the maximum critical temperature. Dashed circles in the two-band regime indicate that $n_{\text {Hall }}(B \rightarrow 0)$ is not the correct carrier density. The gate dependence of the carrier density $n_{2 \mathrm{DEG}}$ has been obtained by integrating the gate capacitance and by matching it to $n_{\text {Hall }}$ in the one-band regime $\left(V_{\mathrm{G}}<-10 V\right)$ (see Methods).

crystal orientations, such as the (110) direction [23] and the (111) direction [26-28]. In this study, we focus on the (110)-oriented $\mathrm{LaAlO}_{3} / \mathrm{SrTiO}_{3}$ heterostructure schematically represented in Fig. 1a. The ability of electrons to hop between two neighbouring Ti lattice sites strongly depends on the spatial directions leading to anisotropic electron-like bands. In this geometry, the $d_{x y}$ band is distinct from the degenerated $d_{x z}$ and $d_{y z}$ bands. Samples were prepared by growing 10 uc-thick $\mathrm{LaAlO}_{3}$ epitaxial layers on $3 \times 3 \mathrm{~mm}^{2}$ (110)-oriented $200 \mu m$-thick $\mathrm{SrTiO}_{3}$ substrate by Pulsed Laser Deposition [23]. A metallic back-gate was then deposited on the backside of the substrate and the $\mathrm{LaAlO}_{3} / \mathrm{SrTiO}_{3}$ heterostructure was inserted into a resonant circuit (Fig. 1b) to perform microwave measurements as described in Methods section and reference [22]. 
After cooling the sample to $450 \mathrm{mK}$, the first positive polarization was applied to a maximum gate voltage $V_{\mathrm{G}}=+50 \mathrm{~V}$ to ensure that no hysteresis would occur upon further gating. This procedure implies that at $V_{\mathrm{G}}=+50 \mathrm{~V}$, the maximum doping has been reached and that the Fermi energy lies at the top of the quantum well [29]. Figure 1c displays the temperature-dependent dc sheet resistance $R$ of the 2-DEG for different gate voltages in the range $[-90 \mathrm{~V},+50 \mathrm{~V}]$. A clear superconducting transition to a zero resistive state is observed for all gate values. The normal state resistance decreases continuously with gate voltage as electrons are added in the 2-DEG. The resulting phase diagram, obtained by plotting the normalized resistance in colour scale as a function of temperature and gate voltage is shown in Fig. 1d. In contrast with the conventional (001)-orientation $[3,12,30]$, superconductivity can not be suppressed by carrier depletion. In the underdoped (UD) regime, the transition temperature weakly increases with $V_{\mathrm{G}}$ to a maximum value of $\simeq 200$ $\mathrm{mK}$ at the optimal doping $V_{\mathrm{G}}^{\mathrm{opt}} \simeq-10 \mathrm{~V}$, before decreasing in the overdoped (OD) regime $\left(V_{\mathrm{G}}>V_{\mathrm{G}}^{\mathrm{opt}}\right)$.

In $\mathrm{SrTiO}_{3}$-based interfaces, the gate dependence of the 2-DEG carrier density can be extracted by combining Hall effect and gate capacitance measurements $[12,31]$. The Hall resistance $R_{\mathrm{H}}$ is linear with the magnetic field $B$ in the UD regime as expected for one-band transport (labelled band 1). In this regime, the carrier density is correctly extracted from the slope of $R_{\mathrm{H}}\left(n_{\mathrm{Hall}}=\frac{B}{e R_{\mathrm{H}}}\right)$. This is not the case in the OD regime $\left(V_{\mathrm{G}} \gtrsim V_{\mathrm{G}}^{\mathrm{opt}}\right)$, where $R_{\mathrm{H}}$ is no longer linear with $B$ because of the presence of an additional population of electrons with a higher mobility (Supplementary Figure 1). Such behaviour suggests that a one-band to two-band transition occurs for a certain gate voltage. In this case, $n_{\text {Hall }}$ measured in the $\mathrm{B} \rightarrow 0$ limit doesn't represent the carrier density (dashed circles in Fig. 1e). The true variation of the 2-DEG carrier density $n_{2 \mathrm{DEG}}$ over the entire phase diagram, can be determined by plotting the charging curve of the gate capacitor which extrapolates $n_{\text {Hall }}$ in the OD regime (Fig. 1e) (see Methods). The new band (band 2) filling threshold, i.e. the Lifshitz transition, corresponds to the gate value where $n_{\text {Hall }}$ deviates from $n_{2 \mathrm{DEG}}[12,31]$. Interestingly, this transition occurs at the optimal doping point $\left(V_{\mathrm{G}}^{\mathrm{opt}} \simeq-10 \mathrm{~V}\right)$, which establishes a correlation between the OD superconducting regime and the two-band transport regime observed in the normal state. However, the significant decrease of the transition temperature in this regime also indicates that the filling of band 2 is detrimental for superconductivity.

We further investigated the superconducting properties of the (110)-oriented $\mathrm{LaAlO}_{3} / \mathrm{SrTiO}_{3}$ interfaces by measuring their superfluid stiffness $J_{s}$, which characterizes the phase rigidity of the condensate. This fundamental energy scale is directly related to the imaginary part of the complex 

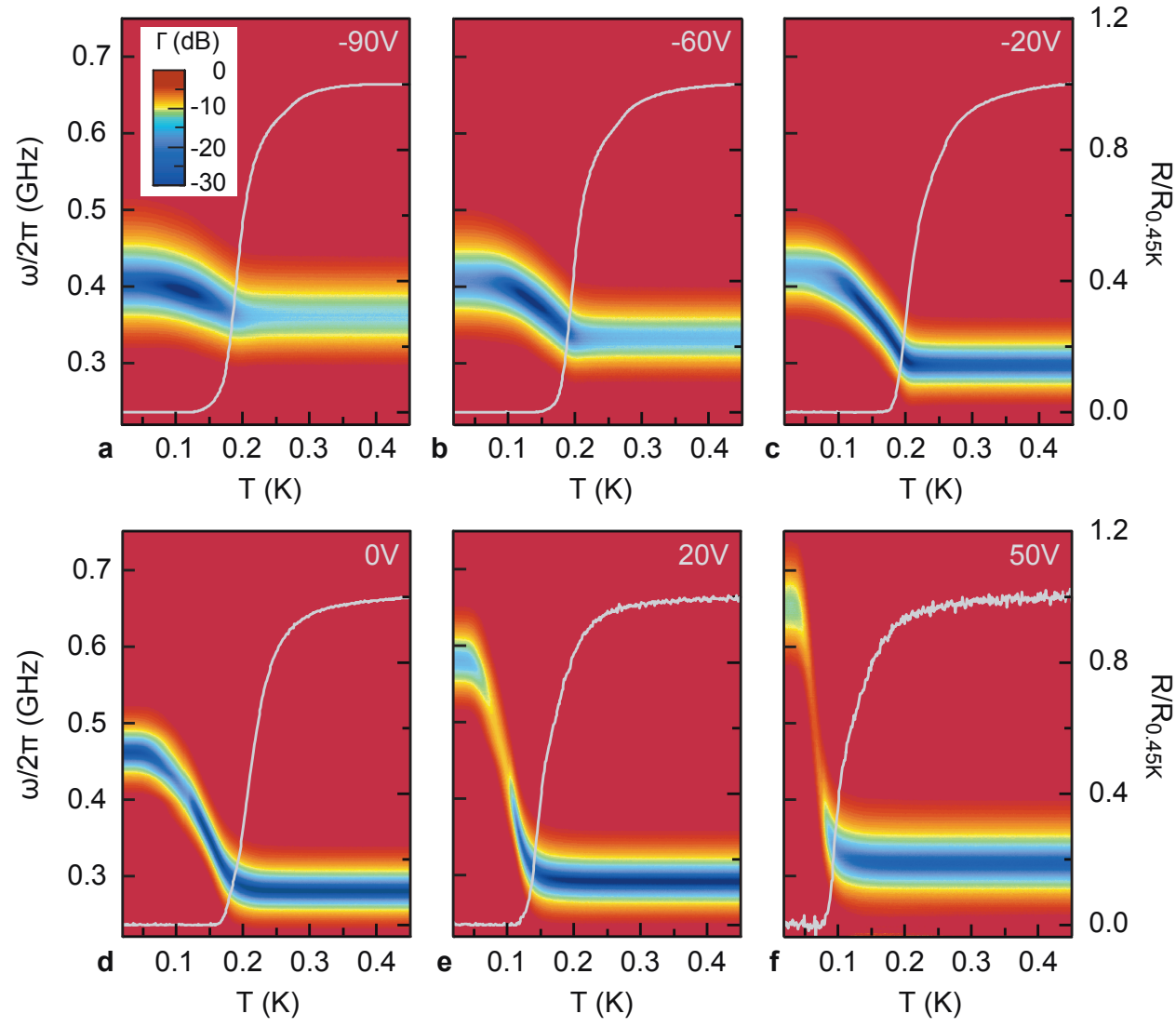

Figure 2: Resonant microwave transport in the superconducting state. (a-f) Magnitude of the reflection coefficient $\Gamma$ in $\mathrm{dB}$ as a function of frequency $\omega$ and temperature for different values of the gate voltage after calibration (see Methods). The temperature dependence of the sheet resistance $\mathrm{R}$, normalized by the normal state resistance $\mathrm{R}_{0.45 K}$ taken at $\mathrm{T}=450 \mathrm{mK}$, is shown on the right axis.

conductivity of the superconductor $\sigma_{2}(\omega)$ at finite frequency $[32,33]$

$$
J_{s}=\frac{\hbar^{2} \sigma_{2}(\omega) \omega}{4 e^{2}}=\frac{\hbar^{2}}{4 e^{2} L_{\mathrm{k}}}
$$

where $L_{\mathrm{k}}$ is the kinetic inductance of the superconductor due of the inertia of the Cooper pairs. In the normal state $\left(T=0.45 \mathrm{~K}>T_{c}\right), \sigma_{2}(\omega)=0$ and the sample circuit described in Fig. $1 \mathrm{~b}$ resonates at the frequency $\omega_{0}=\frac{1}{\sqrt{L_{1} C_{\text {sto }}}}$, which can be determined by measuring the reflection coefficient of the sample circuit $\Gamma(\omega)=\frac{A^{\text {in }}}{A^{\text {out }}}$ (see Methods section). The resonance manifests itself as an absorption dip in the magnitude of $\Gamma(\omega)$ accompanied by a $2 \pi$ phase shift (Supplementary Figure 4) [22]. Figure 2 shows the temperature dependence of $\Gamma(\omega)$ after calibration for a selection of gate voltages spanning the UD (up panels) and OD (down panels) regimes (see Methods). In the superconducting state, the 2-DEG conductance acquires an imaginary part $\sigma_{2}(\omega)=\frac{1}{L_{\mathrm{k}} \omega}$ that generates a shift of $\omega_{0}$ towards high frequencies since the total inductance of the circuit becomes $L_{\text {tot }}(T)=\frac{L_{1} L_{\mathrm{k}}(T)}{L_{1}+L_{\mathrm{k}}(T)}$. The superfluid stiffness $J_{s}(T)$ is thus extracted from the resonance shift for all 
gate values using Eq. (1). The depth of the resonance is controlled by the dissipation of the sample circuit that evolves with temperature (see Methods section). However, $L_{\mathrm{k}}(T)$ is the only relevant quantity to evaluate $J_{s}(T)$.

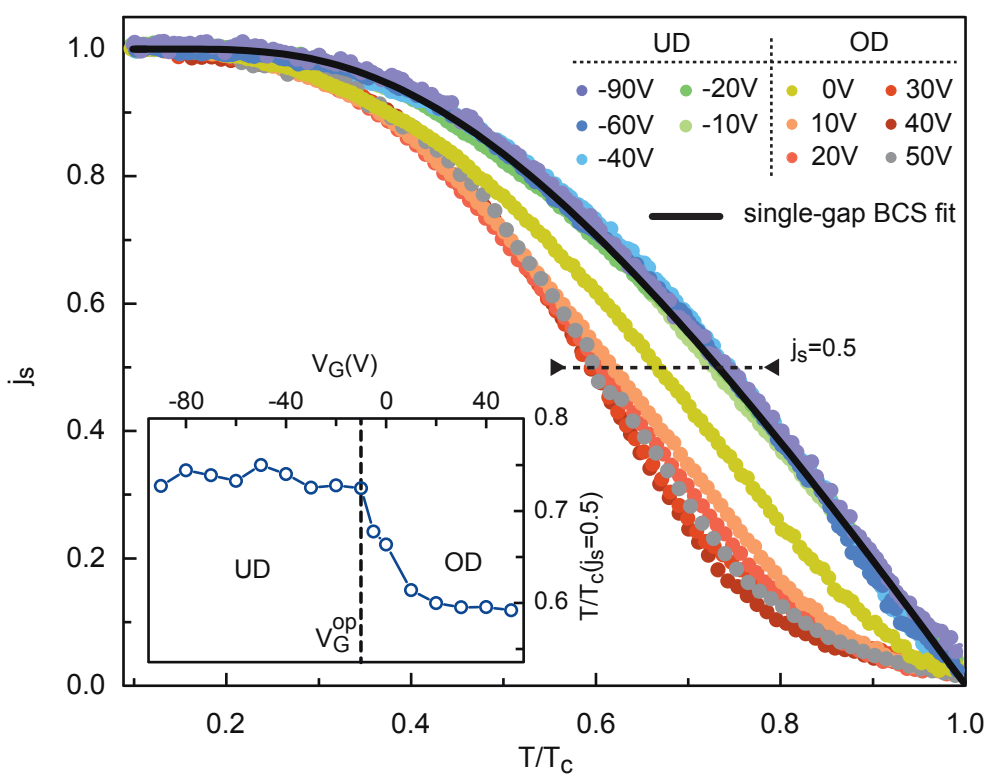

Figure 3: Superfluid stiffness in the underdoped (UD) and overdoped (OD) regimes. Normalized superfluid stiffness $j_{s}(T)=\frac{J_{s}(T)}{J_{s}(0)}$ as a function of the reduced temperature $\frac{T}{T_{c}}$ for different gate voltages spanning the UD and OD regimes. The value of $T_{c}$ used in the reduced temperature is the one extracted from the fit of Fig 4 . In the UD regime, all the curves are superimposed and follow a single-gap BCS behavior (dashed line). In the OD regime, the temperature dependence of the $j_{s}$ curves is strongly modified. The absolute value of $J_{s}$ at $T \simeq 0$ as a function of $V_{\mathrm{G}}$ is shown in Supplementary Figure 5 . (Inset) Reduced temperature $\frac{T}{T_{c}}$ corresponding to $j_{s}=0.5$ as a function of $V_{\mathrm{G}}$. Whereas the values are constant in the UD regime, an abrupt decrease takes place at the Liftshitz transition.

To calculate the superfluid stiffness in multi-band superconductors, Kogan et al. developed a selfconsistent BCS approach based on the quasi-classical Eilenberger weak coupling formalism [34, 35] (Supplementary note 1). For each band, the temperature dependence of the normalized stiffness $j_{s}(T)=\frac{J_{s}(T)}{J_{s}(0)}$ can be derived from the BCS superconducting gap energy $\Delta(T)[34]$

$$
j_{s}=\delta^{2} \sum_{n=0}^{\infty}\left[\delta^{2}+(n+1 / 2)^{2}\right]^{-3 / 2}
$$

where $\delta=\frac{\Delta(T)}{2 \pi k_{B} T}$ is the dimensionless gap energy and $n$ is an integer that defines the Matsubara frequencies $\hbar \omega=\pi T(2 n+1)$. Although Eq. (2) is derived in a clean limit, the temperature dependence of $j_{s}$ on $\Delta(T)$ is only marginally affected by the presence of disorder (Supplementary Note 1). In Figure 3 we compare the temperature dependence of $j_{s}$ in the UD and OD regions of the phase diagram. Two distinct behaviors can be identified. In the UD regime, all the curves overlap 

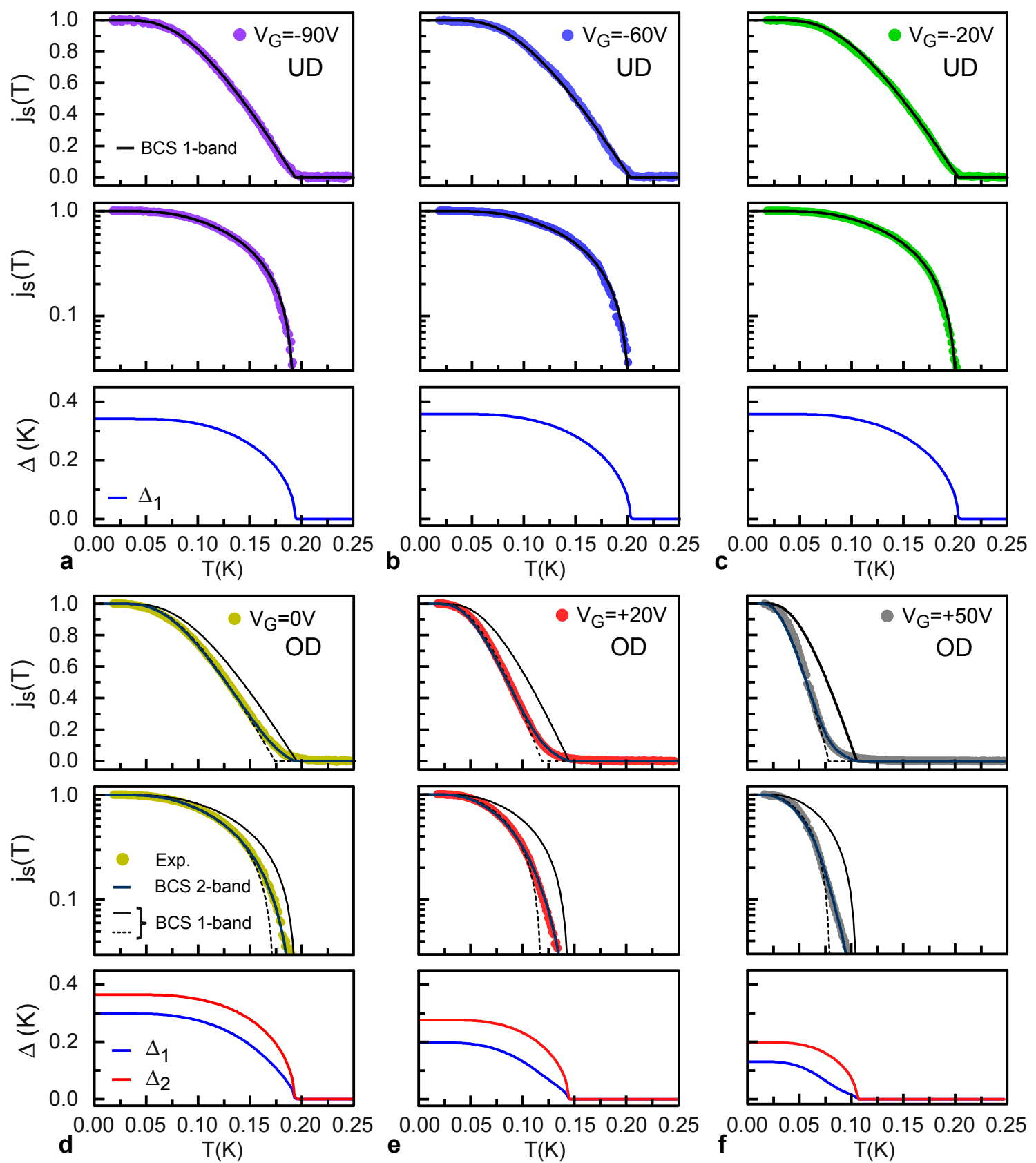

Figure 4: Single-condensate to two-condensate superconductivity transition in the superfluid stiffness. Temperature dependence of $j_{s}$ (symbols) in linear scale (upper panels) and logarithmic scale (middle panels) for three gate voltages in the UD regime $\left((\mathbf{a}) V_{\mathrm{G}}=-90 \mathrm{~V},(\mathbf{b}) V_{\mathrm{G}}=-60 \mathrm{~V}\right.$ and $\left.(\mathbf{c}) V_{\mathrm{G}}=-20 \mathrm{~V}\right)$ and three gate voltages in the OD regime $\left((\mathbf{d}) V_{\mathrm{G}}=0 \mathrm{~V}\right.$, (e) $V_{\mathrm{G}}=20 \mathrm{~V}$ and $(\mathbf{f}) V_{\mathrm{G}}=50 \mathrm{~V}$ ). In the UD regime, $j_{s}(T)$ is fitted using the single-band model (black line) assuming in Eq. (2) the gap energy $\Delta_{1}(T)$ shown in the lower panel for each gate voltage. In the OD regime, $j_{s}(T)$ is fitted using the two-band model (blue line) corresponding to Eq. (3), assuming in Eq. (2) the gap energies $\Delta_{1}(T)$ and $\Delta_{2}(T)$ shown in the lower panel for each gate voltage. Coupling constants are given in Supplementary Figure 7. Two attempts to fit $j_{s}(T)$ in the OD regime with different $T_{c}$ using a single-band model are also shown (black and dashed lines). The fit at optimal doping $V_{\mathrm{G}}^{\mathrm{opt}}=-10 \mathrm{~V}$ is shown in Supplementary figure 6. 
each other and follow a single-gap BCS behavior (Eq. 2) in consistency with the single band Hall effect reported in Fig. 1e. On the other hand, in the OD regime, $j_{s}$ shows a strong deviation with respect to the single-gap BCS behavior. In particular, a change in curvature and a prominent tail appear for $j_{s}<0.5$. The inset of Fig. 3 emphasizes this difference in trend by showing the reduced temperature $\frac{T}{T_{c}}$ corresponding to $j_{s}=0.5$ as a function of gate voltage. Whereas this value is constant in the UD regime, it drops abruptly at $V_{\mathrm{G}}^{\mathrm{opt}}$ and further decreases in the OD regime. This result clearly suggests that a transition between single-condensate superconductivity and two-condensate superconductivity occurs at $V_{\mathrm{G}}^{\mathrm{opt}}$ due to the filling of band 2 as anticipated from Hall effect (Fig. 1e). To calculate the superfluid stiffness in the OD regime, the two superconducting gaps $\Delta_{1}(T)$ and $\Delta_{2}(T)$ are first self-consistently calculated by introducing the intraband $\left(\lambda_{11}, \lambda_{22}\right)$ and interband $\left(\lambda_{12}, \lambda_{21}\right)$ coupling constants (Supplementary Note 1). Then, the temperature dependence of the normalized stiffness for each band $j_{s 1}$ and $j_{s 2}$ is obtained by introducing the corresponding gaps $\Delta_{1}$ and $\Delta_{2}$ in Eq (2). The total normalized stiffness is the sum of the contributions of each band [34]

$$
j_{s}=\gamma j_{s 1}+(1-\gamma) j_{s 2}
$$

where the $\gamma$ coefficient accounts for the weight of each superfluid condensate. A systematic fitting procedure based on this approach was applied to $j_{s}$. As shown in Fig. 4, a very good agreement is obtained between the experimental data and the BCS model both in the UD regime (panels a,b and c) and the OD regime (panels $\mathrm{d}$,e and $\mathrm{f}$ ). In particular, the transition between single-gap and two-gap superconductivity is clearly visible both in linear scale (up sub-panels) and logarithmic scale (middle sub-panels). In the OD regime, the change in curvature and the tail in $j_{s}(T)$ curves are well described by the model considering a weak interband coupling $\left(\left|\lambda_{12(21)}\right|<<\lambda_{11(22)}\right)$. While the temperature dependence of the largest gap $\left(\Delta_{2}\right)$ remains very close to that of a single BCS gap, the coupling generates a change in the curvature of the smallest gap $\left(\Delta_{1}\right)$ near $T_{c}$, which is further reflected in $j_{s}(T)$. The fits imply that the band with the smallest gap and the tail contributes to the total superfluid stiffness with the strongest weight. According to the band hierarchy in the quantum well, this must correspond to band 1, which is mainly filled. Fig. 5c summarizes the values of the superconducting gap energies and the stiffness weight $\gamma$ in the phase diagram extracted from the fitting procedure. In the UD regime, $\Delta_{1}(0)$ very weakly increases with $V_{\mathrm{G}}$, which is consistent with BCS theory since for a $2 \mathrm{D}$ superconductor, $T_{c}$ is in principle carrier density independent. In the OD regime, band 2 is also filled at the Lifshitz transition, and a second superconducting gap $\Delta_{2}\left(>\Delta_{1}\right)$ opens at the Fermi surface. As expected, the $\gamma$ coefficient decreases with gate voltage in this regime as more electrons populate the second band.

Arguing that superconductivity in $\mathrm{SrTiO}_{3}$-based interfaces has likely the same origin than that of bulk $\mathrm{SrTiO}_{3}$, early reports of a distinctive double-gap structure in the tunneling density of states 

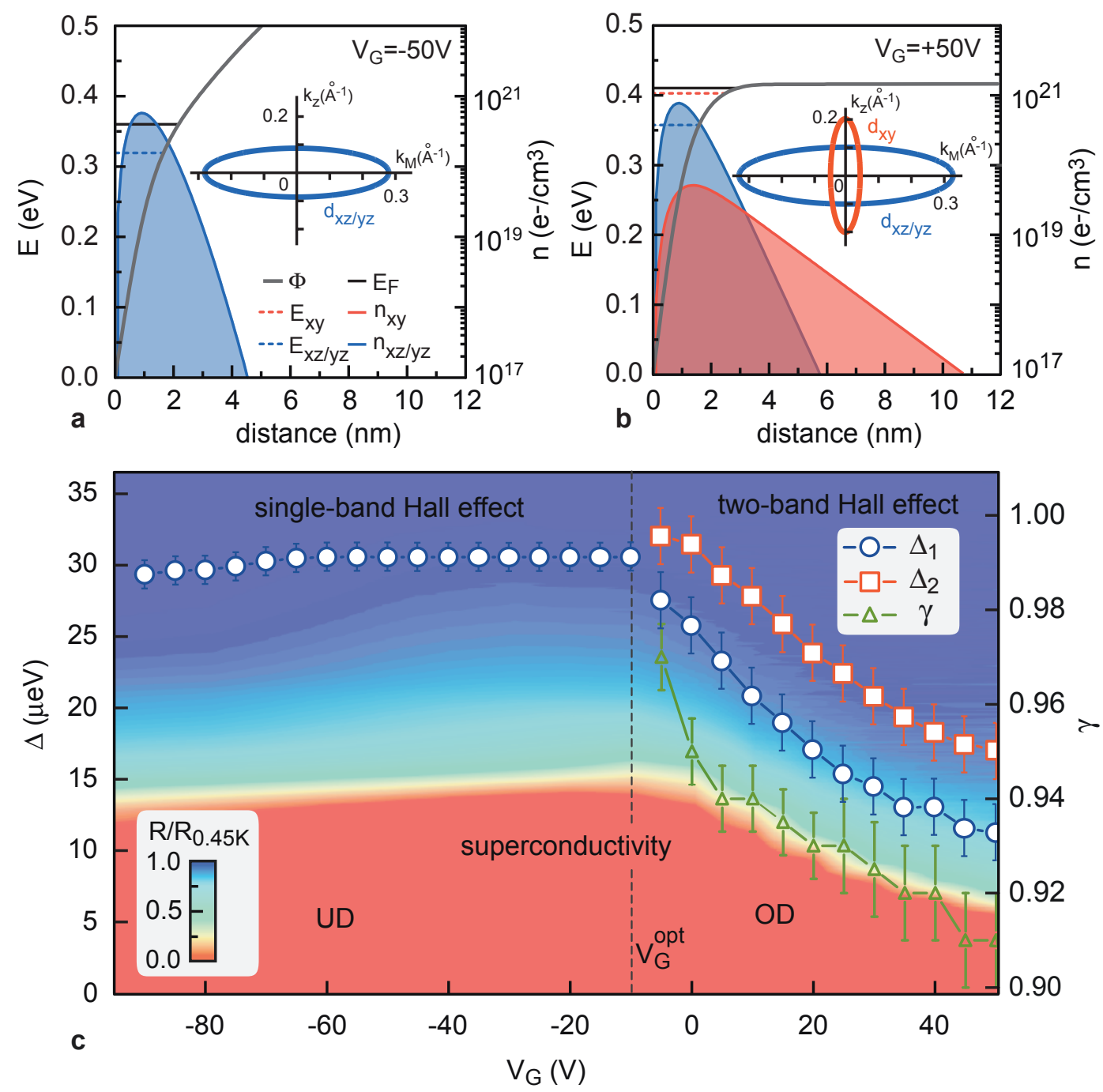

Figure 5: Superconducting phase diagram. a,b) Numerical simulations of the band structure in the (110)-oriented interface using self-consistent Poisson-Schrödinger equations for two doping regimes : $V_{\mathrm{G}}=-50 \mathrm{~V}$ (panel a) and $V_{\mathrm{G}}=+50 \mathrm{~V}$ (panel b, same legend as in panel a). The confinement potential $\Phi$ in energy scale as a function of the distance to the interface, the Fermi energy $\left(E_{F}\right)$ and the energies of the different $t_{2 g}$ bands $\left(E_{x y}, E_{x z / y z}\right)$ are shown in the left axis while the 3D carrier densities associated to each band $\left(n_{x y}, n_{x z / y z}\right)$ are shown in the right axis. The $2 \mathrm{D}$ carrier density used in the simulation corresponds to the one reported on Fig. 1e, i. e. $n \simeq 0.7510^{14} \mathrm{e} / \mathrm{cm}^{2}$ at $V_{\mathrm{G}}=-50 \mathrm{~V}$ and $n \simeq 1.110^{14} \mathrm{e} / \mathrm{cm}^{2}$ at $V_{\mathrm{G}}=+50 \mathrm{~V}$. Insets show the Fermi contours in the $\left(k_{z}([001]), k_{M}([1-10])\right)$ plane for each gate voltage. See Supplementary Information for details on the numerical simulation. c) Gap energies $\Delta_{1}(0)$ and $\Delta_{2}(0)$ ) (left axis), and $\gamma$ coefficient (right axis) extracted from the fitting procedure, plotted as a function of $V_{\mathrm{G}}$ and superimposed on the sheet resistance color map of Fig. 1d. The error bars correspond to the uncertainty on the fitting procedure.

in Nb-doped bulk $\mathrm{SrTiO}_{3}$ above a certain density $\left(\simeq 5 \times 10^{19} \mathrm{~cm}^{-3}\right)[36]$ motivated the theoretical suggestion that multiband superconductivity can also take place in $\mathrm{LaAlO}_{3} / \mathrm{SrTiO}_{3}$ heterostructures $[37,38]$. While the onset of multiple-band occupancy in bulk $\mathrm{SrTiO}_{3}$ has been recently confirmed by the analysis of quantum oscillations in the normal state [39], very recent tunneling [40] and 
a.c. conductivity experiments do not show any evidence for multi-gap superconductivity [41].For heterostructures, only the conventional (001)-orientation has been theoretically considered, but so far, in this case, experiments are consistent with the presence of a single superconducting gap $[22,24,25]$. To discuss the relation between the one-gap to two-gap transition and the filling of the $t_{2 g}$ bands, we numerically simulated the interfacial band structure by self-consistently solving the Poisson-Schrödinger equations (see Supplementary Note 2). Fig. 5a and 5b show the energy of the bands at the centre of the Brillouin zone, inside the interfacial quantum well $\Phi$ (left axis), and the associated spatial distributions of electrons density $\left(n_{x y}, n_{x z / y z}\right)$ for two gate voltages corresponding to the UD and OD regime, respectively. The corresponding Fermi contours are given in the insets of Fig. 5a and 5b. In this crystal orientation, the band hierarchy is reversed with respect to the conventional (001)-orientation $[23,42]$. In the UD regime, only the low-energy degenerated $d_{x z} / d_{y z}$ bands are filled as illustrated in Fig. 5a. These bands, which have a high density of states resulting from an in-plane mass $m_{\|}^{x z, y z} \simeq 2.3 m_{0}$, can host superconductivity $\left(\Delta_{1}, J_{s 1}\right)$, which explains why superconductivity cannot be (or is hardly) suppressed by carrier depletion. This is in contrast with the (001)-orientation, for which the low density of states of the $d_{x y}\left(0.7 m_{0}\right)$ band likely precludes the formation of superconductivity at low doping. At higher doping $\left(V_{\mathrm{G}} \dot{ } V_{\mathrm{G}}^{\mathrm{opt}}\right)$, a $d_{x y}$ band is also filled, which accommodates less than $10 \%$ of the carrier density. As these electrons extend deeper in $\mathrm{SrTiO}_{3}$ substrate, they acquire a mobility sufficiently high to generate a non-linear Hall effect. This band, which has a similar density of states $\left(m_{\|}^{x y} \simeq 3.1 m_{0}\right)$, hosts a second superconducting condensate $\left(\Delta_{2}, J_{s 2}\right)$.

One of the fundamental questions that arises in two-band superconductors is whether the energy gaps in the different bands have the same sign. The temperature dependence of the superfluid stiffness suggests that there are no nodes in the gap that must be constant over each of the two Fermi surfaces. On the other hand, the two superconducting order parameters can have the same sign $\left(s_{++}\right.$-wave pairing) for an attractive interband interaction $\left(\lambda_{12(21)}>0\right)$ or opposite sign $\left(s_{ \pm}\right.$-wave pairing) for a repulsive interband interaction $\left(\lambda_{12(21)}<0\right)$. This issue has been widely discussed in the context of iron-based superconductors, where antiferromagnetic spin fluctuations can provide a repulsive interband pairing channel [43-45], leading to $s_{ \pm}$pairing with two nodeless gaps of opposite signs on the hole and electron pockets. The presence of multiple gaps in these systems has been confirmed by several thermodynamic probes, like e.g. the temperature dependence of the superfluid stiffness [46]. Recently, the sign-changing nature of the order parameter in $\mathrm{Fe}(\mathrm{Se}, \mathrm{Te})$ and FeS was demonstrated by quasiparticle-interference imaging in STM experiments[47, 48]. According to BCS theory, only the positive quantity $\lambda_{12(21)}^{2}$ affects the determination of $T_{c}$. Therefore, the 
coupling with an additional band with a significant density of states should lead to an increase of $T_{c}$, regardless of the sign of $\lambda_{12}[34,37]$. This would then be in contradiction with our findings, where $T_{c}$ decreases soon after that the second band is populated. However, this apparent discrepancy could be reconciled by accounting for impurity scattering in a $s_{ \pm}$superconductor. Indeed, although nodeless single-band superconductivity is essentially insensitive to scattering (provided it is not too strong), in multiband $s_{ \pm}$superconductors scattering processes between bands with opposite-sign gaps are pair-breaking, leading to a suppression of superconductivity [37, 49]. The superconducting (110)-oriented $\mathrm{LaAlO}_{3} / \mathrm{SrTiO}_{3}$ interfaces is in the dirty limit corresponding to $2 \Delta \tau / \hbar \ll 1$ ( $\tau$ is the elastic scattering time). In this view, the $s_{ \pm}$symmetry of the order parameter could explain why the onset of superconductivity in the second band makes the system more sensitive to impurity scattering, leading to the unusual suppression of $T_{c}$ observed in the OD regime. While Ref. [37] provides the starting framework to understand qualitatively this behavior, further investigation is needed to explain the entire gate dependence of $T_{c}$ in this regime.

It is worth noting that the analysis of the temperature dependence of the superfluid stiffness based on Eq. (2) relies mainly on the relative band filling and on the gap values, so it is a robust finding rather insensitive to the presence of disorder. On the other hand, in our fitting procedure the suppression of the gaps and $T_{c}$ in the OD regime can only be reproduced by an artificial decrease of the superconducting coupling constants $\lambda_{i j}$ which could be understood as the effect of disorder within a multiband model with constant pairing interactions and $s_{ \pm}$gap symmetry[38] (Supplementary Note 1). Although the origin of the repulsive interband coupling in our system remains to be identified, we anticipate that it could be related to the specific $t_{2 g}$ orbitals symmetry of the bands. Finally, we would like to mention that the 110-oriented interface also exhibits a Rashba spin-orbit coupling, which hasn't been included in our analysis [23]. In contrast with the (001)-orientation, it is found to be rather gate voltage independent [23] and we therefore do not expect that a possible competition, e.g. between superconductivity and Rashba interaction, could explain the observed anomalous behavior of the gaps.

According to the typical gap energies reported in Fig. 5c, the transition from single-condensate to two-condensate superconductivity in the (110)-oriented $\mathrm{LaAlO}_{3} / \mathrm{SrTiO}_{3}$ interface, can be confirmed by direct tunnelling spectroscopy using planar junctions or STM spectroscopy. However, the relative sign must be investigated using of a phase-sensitive experiment such as a Josephson experiment for example [50]. We also mention that a similar situation can occur in the conventional (001)-oriented interface whose superconducting phase diagram $T_{c}\left(V_{\mathrm{G}}\right)$ displays a characteristic 
dome-shape [3, 12, 51]. Although tunnelling spectroscopy mainly reveals a single gap [24], we cannot exclude that the decrease of $T_{c}$ in the OD regime could originate from a repulsive coupling with a reversed-sign superconducting gap that opens in a high-energy $d_{x y}$ replica band [38]. Because $N(0)$ is weak for the $d_{x y}$ bands in this orientation, interband scattering can contribute to the reduction of $T_{c}$ but the gap may not be easily visible in either tunnelling spectra nor in superfluid stiffness.

[1] Imada, M., Fujimori, A. \& Tokura, Y. Metal-insulator transitions. Rev. Mod. Phys. 70, 1039 (1998).

[2] Ohtomo, A. \& Hwang, H. Y. A high-mobility electron gas at the $\mathrm{LaAlO}_{3} / \mathrm{SrTiO}_{3}$ heterointerface. Nature 427, 423-426 (2004).

[3] Caviglia, A. D., Gariglio, S., Reyren, N., Jaccard, D., Schneider, T., Gabay, M., Thiel, S., Hammerl, G., Mannhart, J. and Triscone, J-M. Electric field control of the $\mathrm{LaAlO}_{3} / \mathrm{SrTiO}_{3}$ interface ground state. Nature 456, 624-627 (2008).

[4] Biscaras, J., Bergeal, N., Kushwaha, A., Wolf, T., Rastogi, A., Budhani, R. C. \& Lesueur, J. Two-dimensional superconductivity at a Mott insulator/band insulator interface $\mathrm{LaTiO}_{3} / \mathrm{SrTiO}_{3}$. Nature Commun. 1, 89 (2010).

[5] Popovic, Z. S., Satpathy, S. \& Martin, R. M. Origin of the two-dimensional electron gas carrier density at the LaAlO 3 on $\mathrm{SrTiO}_{3}$ interface. Phys. Rev. Lett. 101, 256801 (2008).

[6] Delugas, P. et al. Spontaneous 2-dimensional carrier confinement at the n-type $\mathrm{SrTiO}_{3} / \mathrm{LaAlO}_{3}$ interface. Phys. Rev. Lett. 106, 166807 (2011).

[7] Pentcheva, R. \& Pickett, W. Charge localization or itineracy at $\mathrm{LaAlO}_{3} / \mathrm{SrTiO}_{3}$ interfaces: hole polarons, oxygen vacancies, and mobile electrons. Phys. Rev. B 74, 035112 (2006).

[8] Pavlenko, N., Kopp, T., Tsymbal, E., Sawatzky, G. \& Mannhart, J. Magnetic and superconducting phases at the $\mathrm{LaAlO}_{3} / \mathrm{SrTiO}_{3}$ interface: the role of interfacial Ti 3d electrons. Phys. Rev. B 85, 020407(R) (2012).

[9] Scopigno, N., Bucheli, D. Caprara, S., Biscaras, J. Bergeal, N., Lesueur, J. \& Grilli, M. Phase Separation from Electron Confinement at Oxide Interfaces. Phys. Rev. Lett. 116, 026804 (2016).

[10] Salluzzo, M. et al. Orbital Reconstruction and the Two-Dimensional Electron Gas at the $\mathrm{LaAlO}_{3} / \mathrm{SrTiO}_{3} \mathrm{Interface}_{\text {Phys. }}$ Rev. Lett. 102, 166804 (2009).

[11] Seo, S. S. A. et al. Multiple conducting carriers generated in $\mathrm{LaAlO}_{3} / \mathrm{SrTiO}_{3}$ heterostructures. Appl. Phys. Lett. 95, 082107 (2009).

[12] Biscaras, J., Bergeal, N., Hurand, S., Grossetete, C., Rastogi, A. Budhani, R. C., LeBoeuf, D., Proust, C. \& Lesueur, J. Two-dimensional superconductivity induced by high-mobility carrier doping in $\mathrm{LaTiO}_{3} / \mathrm{SrTiO}_{3}$ heterostructures. Phys. Rev. Lett. 108, 247004 (2012).

[13] Kim, J. S., Seo, S. S. A., Chisholm, M. F., Kremer, R. K., Habermeier, H.-U., Keimer, B. \& Lee, H. N. Nonlinear Hall effect and multichannel conduction in $\mathrm{LaTiO}_{3} / \mathrm{SrTiO}_{3}$ superlattices. Phys. Rev. B 82, 201407 (2010).

[14] Ohtsuka, R., Matvejeff, M., Nishio, N., Takahashi, R. \& Lippmaa, M. Transport properties of $\mathrm{LaTiO}_{3} / \mathrm{SrTiO}_{3}$ heterostructures. Appl. Phys. Lett. 96, 192111 (2010).

[15] Caviglia, A. et al. Two-dimensional quantum oscillations of the conductance at $\mathrm{LaAlO}_{3} / \mathrm{SrTiO}_{3}$ interfaces. Phys. Rev. Lett. 105, 236802 (2010).

[16] Ben Shalom, M., Ron, A., Palevski, A. \& Dagan, Y. Shubnikov-de Haas oscillations in $\mathrm{SrTiO}_{3} / \mathrm{LaAlO}_{3}$ interface. Phys. Rev. Lett. 105, 206401 (2010).

[17] Yang, M., Han, K., Torresin, O., Pierre, M., Zeng, S., Huang, Z., Venkatesan, T. V., Goiran, M., Coey, J. M. D., Ariando, 
and Escoffier, W. High-field magneto-transport in two-dimensional electron gas $\mathrm{LaAlO}_{3} / \mathrm{SrTiO}_{3}$. Appl. Phys. Lett. 109, 122106 (2016).

[18] Bardeen, J., Cooper L. N. \& Schrieffer, J. R. Theory of Superconductivity. Phys. Rev. 108, 1175-1204 (1957).

[19] Neville, R. C., Hoeneisen, B. \& Mead, C. A., Permittivity of Strontium Titanate. J. Appl. Phys. 43, 2124 (1972).

[20] Joshua A., Pecker. S, Ruhman, J., Altman, E. \& Ilani, S. A universal critical density underlying the physics of electrons at the $\mathrm{LaAlO}_{3} / \mathrm{SrTiO}_{3}$ interface. Nat. Commun. 3,1129 (2012).

[21] Gariglio, S., Gabay, M. \& Triscone, J.-M. Research Update: Conductivity and beyond at the $\mathrm{LaAlO}_{3} / \mathrm{SrTiO}_{3}$ interface. APL Mater. 4, 060701 (2016).

[22] Singh, G. et al. Competition between electron pairing and phase coherence in superconducting interfaces. Nat. Commun. 9, 407 (2018).

[23] Herranz, G., Singh, G., Bergeal, N., Jouan, A., Lesueur, J., Gázquez, J., Varela, M., Scigaj, M., Dix, N., Sánchez, F. \& Fontcuberta, J., Engineering two-dimensional superconductivity and Rashba spin-orbit coupling in $\mathrm{LaAlO}_{3} / \mathrm{SrTiO}_{3}$ quantum wells by selective orbital occupancy. Nature Commun. 6, 6028 (2015).

[24] Richter, C., Boschker, H., Dietsche, W., Fillis-Tsirakis, E., Jany, R., Loder, F., Kourkoutis, L. F., Muller, D. A., Kirtley, J. R., Schneider, C. W. \& Mannhart, J. Interface superconductor with gap behaviour like a high-temperature superconductor. Nature 502, 528-531 (2013).

[25] Bert, J. A., Nowack, K. C., Kalisky, B., Noad, H., Kirtley, J. R., Bell, C., Sato, H. K., Hosoda, M. Hikita, Y., Hwang, H. Y. \& Moler, K. A. Gate-tuned superfluid density at the superconducting $\mathrm{LaAlO}_{3} / \mathrm{SrTiO}_{3}$ interface. Phys. Rev. B 86, 060503(R) (2012).

[26] Monteiro, A. M. R. V. L., Groenendijk, D. J., Groen, I., de Bruijckere, J., Gaudenzi, R., van der Zant, H. S. J. \& Caviglia A. D. Two-dimensional superconductivity at the (111) $\mathrm{LaAlO}_{3} / \mathrm{SrTiO}_{3}$ interface Phys. Rev. B , 96 020504(R) (2017).

[27] Rout, P. K., Maniv, E. \& Dagan, Y. Link between the Superconducting Dome and Spin-Orbit Interaction in the (111) $\mathrm{LaAlO}_{3} / \mathrm{SrTiO}_{3}$ interface. Phys. Rev. Lett. 119, 237002 (2017).

[28] Davis, S., Huang, Z., Han, K., Ariando, Venkatesan, T. \& Chandrasekhar, V. Superconductivity and Frozen Electronic States at the (111) $\mathrm{LaAlO}_{3} / \mathrm{SrTiO}_{3}$ Interface. Phys. Rev. Lett. 119, 237002 (2017).

[29] Biscaras, J., Hurand, S., Feuillet-Palma, C., Rastogi, A, Budhani, R. C., Reyren, N., Lesne, E., Lesueur, J. \& Bergeal, N. Limit of the electrostatic doping in two-dimensional electron gases of $\mathrm{LaXO}_{3}(\mathrm{X}=\mathrm{Al}, \mathrm{Ti}) / \mathrm{SrTiO}_{3}$. Sci. Rep. 4, 6788 (2014).

[30] Hurand, S., et al. Field-effect control of superconductivity and Rashba spin-orbit coupling in top-gated $\mathrm{LaAlO}_{3} / \mathrm{SrTiO}_{3}$ devices. Sci. Rep. 5, 12751 (2015).

[31] Singh, G., Jouan, A., Hurand, S., Feuillet-Palma, C., Kumar, P., Dogra, A., Budhani, R., Lesueur, J. \& Bergeal, N. Effect of disorder on superconductivity and Rashba spin-orbit coupling in $\mathrm{LaAlO}_{3} / \mathrm{SrTiO}_{3}$ interfaces. Phys. Rev. B 96, 024509 (2017).

[32] Mattis, C. \& Bardeen, J. Theory of the Anomalous Skin Effect in Normal and Superconducting Metals. Phys. Rev. 111, $412(1958)$.

[33] Dressel, M. Electrodynamics of Metallic Superconductors. Adv. Condens. Matter Phys., 2013, 104379 (2013).

[34] Kogan, V. G., Martin, C. \& Prozorov, R. Superfluid density and specific heat within a self-consistent scheme for a two-band superconductor. Phys. Rev. B 80, 014507 (2009).

[35] Kim, H., Tanatar, M. A., Yoo Jang Song, Yong Seung Kwon, Y. S. \& Prozorov, R. Nodeless two-gap superconducting state in single crystals of the stoichiometric iron pnictide LiFeAs. Phys. Rev. B 83, 100502(R) (2011).

[36] Binning, G., Baratoff, A., Hoenig, H. E. \& Bednorz, J. C. Two-Band Superconductivity in Nb-Doped SrTiO 3. Phys. Rev. Lett. 45, 1352 (1980).

[37] Fernandes, R. M., Haraldsen, J. T., Wölfle, P. \& Balatsky, A. V. Two-band superconductivity in doped $\mathrm{SrTiO}_{3}$ films and interfaces. Phys. Rev. B 87, 014510 (2013).

[38] Trevisan, T. V., Schütt, M., Fernandes, R. M. Unconventional multi-band superconductivity in bulk $\mathrm{SrTiO}_{3}$ and $\mathrm{LaAlO}_{3} / \mathrm{SrTiO}_{3}$ interfaces. Phys. Rev. Lett. 121, 127002 (2018). 
[39] X. Lin et al., Critical Doping for the Onset of a Two-Band Superconducting Ground State in $\mathrm{SrTiO}_{3-\delta}$. Phys. Rev. Lett. 112, 207002 (2014).

[40] Swartz A. G., Inoue, H., Merz, T. A., Hikita Y., Raghu, S., Devereaux, T. P., Johnstone, S. \& Hwang, H. Y. Polaronic behavior in a weak-coupling superconductor. PNAS, 115, 1475-1480 (2018).

[41] M. Thiemann et al. Single-gap superconductivity and dome of superfluid density in Nb-doped $\mathrm{SrTiO}_{3}$. Phys. Rev. Lett. 120, 237002 (2018).

[42] Pesquera, D. et al. Two-Dimensional Electron Gases at $\mathrm{LaAlO}_{3} / \mathrm{SrTiO}_{3}$ Interfaces: Orbital Symmetry and Hierarchy Engineered by Crystal Orientation. Phys. Rev. Lett. 113, 156802 (2014).

[43] Mazin, I. I., Singh, D. J., Johannes, M. D. \& Du, M. H. Unconventional Superconductivity with a Sign Reversal in the Order Parameter of $\mathrm{LaFeAsO}_{1-x} \mathrm{~F}_{x}$. Phys. Rev. Lett. 101, 057003 (2008).

[44] Hirschfeld, P. J., Korshunov, M. M. \& Mazin, I. I. Gap symmetry and structure of Fe-based superconductors. Rep. Prog. Phys 74, 124508 (2011).

[45] Wang, F. \& Lee, D.-H. The electron-pairing mechanism of iron-based superconductors. Science 332, 200-204 (2011).

[46] Prozorov, R. \& Kogan, V. G. London penetration depth in iron-based superconductors. Rep. Prog. Phys 74, 124505 (2011).

[47] Hanaguri, T., Niitaka, S., Kuroki, K. \& Takagi H. Unconventional s-Wave Superconductivity in Fe(Se,Te). Science, 328, 474-476 (2010).

[48] Sprau, P. O. et al. Discovery of orbital-selective Cooper pairing in FeSe. Science, 357, 75-80 (2017).

[49] Kogan, V. G. \& Prozorov, R. Interband coupling and nonmagnetic interband scattering in \pm s superconductors. Phys. Rev. B 93, 224515 (2016).

[50] Chen, C.-T., Tsuei, C. C., Ketchen, M. B., Ren, Z.-A. \& Zhao, Z. X. Integer and half-integer flux-quantum transitions in a niobium-iron pnictide loop. Nat. Phys. 6, 260 (2010).

[51] Bell, C., Harashima, S., Kozuka, Y., Kim, M., Kim, B. G., Hikita, Y. \& Hwang, H. Y., Dominant Mobility Modulation by the Electric Field Effect at the $\mathrm{LaAlO}_{3} / \mathrm{SrTiO}_{3}$ Interface. Phys. Rev. Lett. 103, 226802 (2009).

[52] Hemberger, J., Lunkenheimer, P., Viana, R., Bohmer, R. and Loidl, A., Phys. Rev. B 52, 13159 (1995).

\section{Acknowledgments}

We acknowledge K. Behnia and J. Lorenzana for useful discussions. This work was supported by the Région Ile-de-France in the framework of CNano IdF, OXYMORE and Sesame programs, by CNRS through a PICS program (S2S) and ANR JCJC (Nano-SO2DEG). This work was supported by the Spanish MAT2017-85232-R, MAT2014-56063-C2-1-R, Severo Ochoa SEV-2015-0496 grant, and the Generalitat de Catalunya (2017 SGR 1377). This work was supported by the Italian MAECI under the Italia-India collaborative project SUPERTOP-PGR04879. The Authors acknowledge received funding from the project Quantox of QuantERA ERA-NET Cofund in Quantum Technologies (Grant Agreement N. 731473) implemented within the European Union's Horizon 2020 Program. The authors also acknowledge the COST project Nanoscale coherent hybrid devices for superconducting quantum technologies-Action CA16218.

\section{Author contributions}

N.B. conceived and directed the project. G. S. and A. J. performed the measurements under the supervision of N.B.. Samples were fabricated by G. H., M. S. and F. S.. G. S., A. J. and N. B. carried out the analysis of the results and wrote the article with the help of L. B. and J. L.. G. S., G. H., S. C., M. G., C. F-P.. contributed to discussions of the results and commented the final manuscript.

\section{Competing interests:}

The authors declare no competing financial interests.

\section{Additional information}


Supplementary Information accompanies this paper.

Correspondence and requests for materials should be addressed to N. B.

\section{Methods}

\section{Sample preparation and resonant circuit.}

The 10 u.c. thick $\mathrm{LaAlO}_{3}$ film was grown by pulsed laser deposition $(\lambda=248 \mathrm{~nm})$ monitored by reflection high-energy electron diffraction (RHEED). The substrate was heated from room temperature to deposition temperature $\left(850^{\circ} \mathrm{C}\right)$ in an oxygen partial pressure $\mathrm{P}\left(\mathrm{O}_{2}\right)=0.1$ mbar. During deposition, the $\mathrm{LaAlO}_{3}$ was grown under a pressure $\mathrm{P}\left(\mathrm{O}_{2}\right)=10^{-4}$ mbar and a $1 \mathrm{~Hz}$ repetition rate, with laser pulse energy of around $26 \mathrm{~mJ}$. At the end of the deposition, the sample was cooled down in oxygen rich atmosphere to minimize the formation of oxygen vacancies that could lead to extrinsic mechanisms of conduction. More specifically, the samples were cooled from $\mathrm{T}=850^{\circ} \mathrm{C}$ to $750^{\circ} \mathrm{C}$ under a pressure $\mathrm{P}\left(\mathrm{O}_{2}\right)=0.3$ mbar and under $\mathrm{P}\left(\mathrm{O}_{2}\right)$ $=200$ mbar from $\mathrm{T}=750^{\circ} \mathrm{C}$ down to room temperature, including a dwell time of 1 hour at $600^{\circ} \mathrm{C}$. After the growth, a weakly conducting metallic back-gate was deposited on the backside of the $200 \mu m$-thick substrate. The $\mathrm{LaAlO}_{3} / \mathrm{SrTiO}_{3}$ heterostructure was inserted in a microwave circuit board between the central strip of a coplanar waveguide transmission line and its ground to perform microwave measurements as described in reference [22]. The high sheet resistance $(\sim 100 \mathrm{k} \Omega)$ guarantees that the gate doesn't short-circuit the 2-DEG at microwave frequencies. The Surface Mounted microwave Devices (SMD), which include resistor $R_{1}$ and inductor $L_{1}$, were added to form a parallel RLC resonant circuit, where the capacitance $C_{\mathrm{STO}}$ is the intrinsic capacitance of the $\mathrm{SrTiO}_{3}$ substrate (Fig. 1b).

\section{Hall effect and gate capacitance.}

The (110)-oriented $\mathrm{LaAlO}_{3} / \mathrm{SrTiO}_{3}$ interface displays a Hall effect linear with magnetic field for $\mathrm{V}_{\mathrm{G}}<V_{\mathrm{G}}^{\mathrm{opt}}=-10 \mathrm{~V}$ as expected for single band transport. For $\mathrm{V}_{\mathrm{G}}>V_{\mathrm{G}}^{\mathrm{opt}}$, a nonlinear Hall effect is observed due to the filling of a second band (Supplementary Figure 1) [12, 31]. The carrier density $n_{\text {Hall }}=\frac{B}{e R_{\text {Hall }}}$, reported in Fig.1e of the main text, which is extracted from $R_{\text {Hall }}$ in the limit $\mathrm{B} \rightarrow 0$, is only meaningful in the linear regime. However, the correct variation of carrier density with $V_{\mathrm{G}}$ can be retrieved from the charging curve of the capacitor given by the integral of the gate capacitance $C\left(V_{\mathrm{G}}\right)$ :

$$
n_{2 \mathrm{DEG}}\left(V_{\mathrm{G}}\right)=n_{\text {Hall }}\left(V_{\mathrm{G}}=-90 V\right)+\frac{1}{e A} \int_{-120}^{V_{\mathrm{G}}} C\left(V_{\mathrm{G}}\right) d V
$$

where $A$ is the area of the sample. As shown in Figure 1 of the main text, $n_{2 \text { DEG }}$ matches $n_{\text {Hall }}$ 
in the single-band regime $\left(V_{\mathrm{G}}<V_{\mathrm{G}}^{\mathrm{opt}}\right)$ and extrapolates the curve in the two-band regime.

\section{Reflection coefficient measurement and calibration}

A directional coupler is used to guide the microwave signal from the input port to the sample through a bias-tee, and to separate the reflected signal which is amplified by a low-noise cryogenic HEMT amplifier before reaching the output port [22]. The complex transmission coefficient $S_{21}(\omega)$ between the two ports is measured with a vector network analyzer. Standard microwave network analysis relates the reflection coefficient of the RLC sample circuit $\Gamma(\omega)$ to the measured $S_{21}(\omega)$, through complex error coefficients that can be determined by a calibration (Supplementary Figure 2). In this experiment, the microwave set-up was calibrated by using as references, the impedances $Z_{\mathrm{c}}$ of the sample circuit in the normal state of the 2-DEG at $T=450 \mathrm{mK}$ for three gate voltages $\left(V_{\mathrm{G}}=-90 \mathrm{~V}, V_{\mathrm{G}}=0 \mathrm{~V}\right.$ and $\left.V_{\mathrm{G}}=50 \mathrm{~V}\right)$. The complex reflection coefficient $\Gamma(\omega)$ is given by

$$
\Gamma(\omega)=\frac{A^{\text {out }}(\omega)}{A^{\text {in }}(\omega)}=\frac{Z_{c}(\omega)-Z_{0}}{Z_{c}(\omega)+Z_{0}}
$$

where $A^{\text {in }}$ and $A^{\text {out }}$ are the complex amplitudes of incident and reflected waves and $Z_{0}=50 \Omega$ is the characteristic impedance of the CPW transmission line. A reflection measurement gives therefore a direct access to the circuit impedance $Z_{c}(\omega)$. At the resonance frequency, $Z_{c}$ becomes purely real and energy is dissipated in the resonant circuit, leading to an absorption dip in the magnitude of $\Gamma(\omega)$ accompanied by a $2 \pi$ phase shift. Supplementary Figure 4 shows the magnitude of the reflection coefficient $\Gamma$ as a function of frequency and gate voltage at $\mathrm{T}=450$ $\mathrm{mK}$. The resonance manifests itself as a dip in the magnitude of $\Gamma(\omega)$ accompanied by a $2 \pi$ phase shift. The value of $C_{\text {sto }}$ which is gate dependent because of the electric-field-dependence of the dielectric constant of the $\mathrm{SrTiO}_{3}$ substrate [52] can be determined from the resonance

frequency $\omega_{0}=\frac{1}{\sqrt{L_{1} C_{s t o}}}$ for each value of the gate voltage. The depth of the resonance is controlled by the dissipation of the circuit which includes $R_{1}$ and the 2-DEG contribution. This latter can be described by the real part of the conductance $\sigma_{1}$. However, $C_{\text {sto }}$ has also an imaginary part accounting for $\mathrm{SrTiO}_{3}$ losses, which contributes to the total dissipation. These losses don't interfere in the calculation of $J_{s}$ since they don't affect the resonance frequency $\omega_{0}$, but they make the determination of $\sigma_{1}(T)$ (not presented here) more difficult. To maximize the depth of the resonance and make it clearly visible in all the gate and temperature ranges, the value of resistor $R_{1}$ was chosen such that the total dissipation of the circuit is always close to $Z_{0}=50 \Omega$. Supplementary Figure 3 shows a comparison between raw data and calibrated data in the normal state and superconducting state. As seen from panels c and d, the calibration doesn't 
introduce artefacts in the extraction of $J_{s}(T)$.

\section{Data availability}

All data that support the findings of this study are available from the corresponding authors upon reasonable request. 